\title{
Frontal information flow and connectivity in psychopathy
}

Yaling Yang, Adrian Raine, Anand A. Joshi, Shantanu Joshi, Yu-Teng Chang, Robert A. Schug, David Wheland, Richard Leahy and Katherine L. Narr

\section{Summary}

Despite accumulating evidence of structural deficits in individuals with psychopathy, especially in frontal regions, our understanding of systems-level disturbances in cortical networks remains limited. We applied novel graph theorybased methods to assess information flow and connectivity based on cortical thickness measures in 55 individuals with psychopathy and 47 normal controls. Compared with controls, the psychopathy group showed significantly altered interregional connectivity patterns. Furthermore, bilateral superior frontal cortices in the frontal network were identified as information flow control hubs in the psychopathy group in contrast to bilateral inferior frontal and medial orbitofrontal cortices as network hubs of the controls. Frontal information flow and connectivity may have a significant role in the neuropathology of psychopathy.

\section{Declaration of interest}

None.
People with a diagnosis of psychopathy are characterised by shallow affect, lack of empathy or guilt, egocentricity, impulsiveness and poor behavioural control. Several neuroimaging studies report abnormalities in frontal lobe regions that are thought to contribute to impaired moral decision-making, emotional dysregulation and social dysfunction in such individuals. ${ }^{1-3}$ Despite progress in identifying regional morphological abnormalities, how these structural deficits relate to the complex organisation of cortical networks remains unknown. In this study we applied methods based on graph theory ${ }^{4,5}$ to investigate the anatomical connectivity of the cortex in 55 individuals with high scores on Hare's Psychopathy Checklist - Revised and 48 healthy controls. ${ }^{1,6}$ In addition, we examined small-world network properties to determine whether topological characteristics of anatomical network organisation were altered in individuals with psychopathy.

\section{Method}

Participants were recruited from the Greater Los Angeles area and demographic, cognitive and diagnostic characteristics were obtained as described previously. ${ }^{1}$ Groups did not differ in age, gender, ethnicity or handedness (all $P>0.05$ ). For each participant, structural magnetic resonance imaging (MRI) data were collected on a $3 \mathrm{~T}$ Siemens Trio scanner (Siemens, Erlangen, Germany); repetition time was $1380 \mathrm{~ms}$, echo time $2.6 \mathrm{~ms}$, voxel size $1 \mathrm{~mm}^{3}$. After scalp stripping, tissue classification, surface extraction and cortical parcellation with manual editing of errors using FreeSurfer (version 5.1.0) processing streams, ${ }^{7}$ the whole brain cortical surface was segmented into 34 regions of interest (ROIs) using the Desikan template and the mean cortical grey matter thickness of each ROI was estimated for each participant. ${ }^{7,8}$ Structural connectivity was estimated by calculating the partial correlation between mean thickness values for each pair of regions for each individual using a modified partial correlation algorithm (see online supplement), and the two areas were defined as anatomically connected if the correlation was significant. ${ }^{8}$ The resulting partial correlation matrices were thresholded to construct an undirected graph representing the true structural connectivity of the network for each group and bootstrapping procedures established whether correlations in the network differed significantly between the two groups. To correct for multiple comparisons, a false discovery rate (FDR) test was performed using a $q$ value of 0.01 . Post hoc analyses focused on the a priori frontal sub-network including 11 ROIs (online Fig. DS1(b)), which are all the frontal regions from the original 34 ROIs estimated. Analyses were then performed to derive the topological parameters for this sub-network including small-world properties (i.e. mean network characteristic path length and clustering coefficient) and nodal centrality (i.e. betweenness of each node). ${ }^{4,5,8}$

\section{Results}

The group comparison matrix is shown in Fig. DS1(a), and interregional partial correlation matrices for cortical thickness measurements obtained from both groups are shown in online Fig. DS2. The group comparison matrix showed significant differences in interregional correlations between groups across several regions of the brain that survived the FDR correction with $q=0.01$ (equivalent to $P<0.0005$ ). For the frontal sub-network, the psychopathy group exhibited weaker intrahemisphere interregional correlations in the right side (lower right quadrant of Fig. DS1(b)) compared with controls.

Topological measurements of frontal sub-networks were derived from the interregional correlation matrices (Fig. DS2) and are outlined in online Table DS1. These measurements revealed that the values of clustering coefficients $(0.16)$, local efficiency (0.26) and characteristic path length $(0.17)$ in the right frontal cortex in individuals with psychopathy are reduced compared with controls $(0.25,0.48$ and 0.25 respectively) (Table DS1). We further evaluated the betweenness (information flow) of each frontal node by identifying hubs with betweenness values twice the average betweenness of the network. The average betweenness value was 46.9 for the psychopathy group and 46.2 for the control group for the frontal network and results showed bilateral superior frontal cortex (left 216, right 158) to be information flow hubs in the psychopathy group, whereas bilateral inferior frontal (left 140, right 144) and medial orbitofrontal cortex (left 132, right 212) were hubs in controls (Table DS1).

\section{Discussion}

Our findings present the first evidence of alterations in information flow and connectivity in individuals with psychopathy. 
Findings are consistent with and extend those of several studies showing structural brain deficits, particularly in the right frontal regions, in individuals who are either psychopathic or antisocial, and support the hypothesis that psychopathy is a consequence of inherited or acquired deficits in frontal brain areas and networks. ${ }^{9}$ We found altered interregional connectivity in the psychopathy group between several regions across the whole brain, including many of the interhemispheric correlations between each pair of the ROIs (i.e. left and right). Findings are consistent with previous reports of abnormal corpus callosum structure in individuals with psychopathy, ${ }^{10}$ which may reflect disturbances in neurodevelopmental processes involving synaptic pruning or myelination. Findings are further supported by evidence of white matter microstructural abnormalities in individuals with psychopathy from previous studies. Using diffusion tensor imaging, Sundram et al found reduced fractional anisotropy in the uncinate and inferior fronto-occipital fasciculus, anterior corona radiata and the anterior limb and genu of the internal capsule, particularly within the right frontal lobe, to be negatively correlated with measures of psychopathy. ${ }^{3}$ The disrupted white matter connectivity in these fibre pathways may contribute to the altered interregional correlations in the psychopathy group and explain the lower clustering coefficients and decreased local efficiency of right frontal networks. On the other hand, increased interregional correlations were observed in some frontal regions, suggesting that certain frontal connectivity may be strengthened in order to compensate for other weakened connectivity (which might be due to abnormalities such as localised grey matter thinning) and which might allow individuals to perform within the normal range in many traditional neuropsychological tasks. ${ }^{11}$

Our results further indicate that the topological components within the frontal network are also different between psychopathic and non-psychopathic samples. A key finding was the difference in network hubs (i.e. brain regions showing important influence over information flow between other regions in the network) between the two groups. As expected based on previously identified cortical networks in normal individuals, ${ }^{8}$ we identified bilateral inferior frontal and medial orbitofrontal cortices as information flow hubs in the frontal network of our control group participants. In contrast, bilateral superior frontal cortices were identified as hubs in the psychopathy group, consistent with previous observations suggesting that the superior frontal regions are largely spared from any prominent structural alteration in psychopathy. ${ }^{1}$ On the other hand, inferior frontal and medial orbitofrontal cortical thickness, areas identified as key hubs in the frontal network of normal participants, has been found to be reduced in individuals with psychopathy. ${ }^{1,2}$ Last, it is worth mentioning that the use of partial correlations to study anatomical brain networks is largely based on the assumption that positive correlations indicate connectivity, as regions connected through fibre tracts are believed to share developmental and maturational influences. ${ }^{12}$ Although the exact biological nature of the cortical thickness partial correlations is still unknown, it has been argued that mutually trophic influences, genetic contributions or common environment-related plasticity may all contribute to the observed interregional correlations. ${ }^{8}$ This is supported by several studies showing that the correlational patterns identified using graph theory analysis methods closely resemble findings from tract-tracing studies. ${ }^{13}$ However, although findings of altered information flow, efficiency and connectivity may imply functional disturbances, future studies are needed to confirm topological relationships between structural and functional networks.
Overall, findings provided initial evidence supporting the argument that alterations in anatomical connection patterns may play a critical part in the neural mechanisms underlying psychopathy. In future work it might help to integrate different imaging modalities (including diffusion tensor imaging and resting state functional MRI) to assess the direct anatomical and functional connections, to further clarify the nature of multilevel brain network disturbances in individuals with psychopathy and to advance our understanding of the complex neuropathology underlying psychopathy.

\footnotetext{
Yaling Yang, Laboratory of Neuro Imaging, Department of Neurology, David Geffen School of Medicine at University of California, Los Angeles (UCLA), California; Adrian Raine, Department of Criminology, Psychiatry and Psychology, University of Pennsylvania, Philadelphia, Pennsylvania; Anand A. Joshi, Biomedical Imaging Research Laboratory, Department of Electrical Engineering, University of Southern California; Shantanu Joshi, Laboratory of Neuro Imaging, Department of Neurology, David Geffen School of Medicine at UCLA; Yu-Teng Chang, Biomedical Imaging Research Laboratory, Department of Electrical Engineering, University of Southern California; Robert A. Schug, Department of Criminal Justice, California State University, Long Beach, California; David Wheland, Richard Leahy, Biomedical Imaging Research Laboratory, Department of Electrical Engineering, University of Southern California; Katherine L. Narr, Laboratory of Neuro Imaging, Department of Neurology, David Geffen School of Medicine at UCLA, California, USA

Correspondence: Yaling Yang, Department of Neurology, David Geffen Schoo of Medicine, University of California, 1721 Speyer Lane, Redondo Beach, Los Angeles, CA 90278, USA. Email: yalingy@usc.edu

First received 7 Dec 2011, final revision 14 May 2012, accepted 29 May 2012
}

\section{References}

1 Yang Y, Raine A, Colletti P, Toga AW, Narr KL. Abnormal temporal and prefrontal cortical gray matter thinning in psychopaths. Mol Psychiatry 2009; 14: $561-2,555$

2 Yang $Y$, Raine A. Prefrontal structural and functional brain imaging findings in antisocial, violent, and psychopathic individuals: a meta-analysis. Psychiatry Res 2009; 174: 81-8.

3 Sundram F, Deeley Q, Sarkar S, Daly E, Latham R, Graig M, et al. White matter microstructural abnormalities in the frontal lobe of adults with antisocial personality disorder. Cortex 2012; 48: 216-29.

4 Rubinov M, Sporns O. Complex network measures of brain connectivity: uses and interpretations. Neuroimage 2010; 52: 1059-69.

5 Bullmore E, Sporns O. Complex brain networks: graph theoretical analysis of structural and functional systems. Nat Rev Neurosci 2009; 10: 186-98.

6 Hare RD. Manual for the Revised Psychopathy Checklist (2nd edn). MultiHealth Systems, 2003

7 Fischl B, Dale AM. Measuring the thickness of the human cerebral cortex from magnetic resonance images. Proc Natl Acad Sci USA 2000; 97 11050-5.

8 He Y, Chen ZJ, Evans AC. Small-world anatomical networks in the human brain revealed by cortical thickness from MRI. Cereb Cortex 2007; 17: 2407-19.

9 Müller JL, Sommer M, Wagner V, Lange $\mathrm{K}$, Taschler $\mathrm{H}$, Röder $\mathrm{CH}$, et al. Abnormalities in emotion processing within cortical and subcortical regions in criminal psychopaths: evidence from a functional magnetic resonance imaging study using pictures with emotional content. Biol Psychiatry 2003; 54: $152-62$.

10 Raine A, Lencz T, Taylor K, Hellige JB, Bihrl S, Lacasse L, et al. Corpus callosum abnormalities in psychopathic antisocial individuals. Arch Gen Psychiatry 2003; 60: 1134-42.

11 Smith SS, Arnett PA, Newman JP. Neuropsychological differentiation of psychopathic and nonpsychopathic criminal offenders. Pers Individ Dif 1992; 13: 1233-43.

12 Bernhardt BC, Chen Z, He Y, Evans AC, Bernasconi N. Graph-theoretical analysis reveals disrupted small-world organization of cortical thickness correlation networks in temporal lobe epilepsy. Cereb Cortex 2011; 21: 2147-57.

13 Lerch JP, Worsley K, Shaw WP, Greenstein DK, Lenroot RK, Giedd J, et al Mapping anatomical correlations across cerebral cortex (MACACC) using cortical thickness from MRI. Neuroimage 2006; 31: 993-1003. 